\title{
BEREND WIERENGA*
}

The author presents a new method for estimating the parameters of the linear learning model. The procedure, essentially a least squares method, is easy to carry out and avoids certain difficulties of earlier estimation procedures. Applications to three different data sets are reported, as well as results from a goodness-of-fit test. A simulation study was carried out to validate the method. The outcomes are compared with those obtained from the minimum chi square estimation method. The results of the new method appear to be satisfactory.

\section{A Least Squares Estimation Method for the Linear Learning Model}

\section{INTRODUCTION}

The linear learning method (LLM) has proved to be a successful tool for modeling consumer choice phenomena. A broad spectrum of applications of this model, with respect to both brand choice and store choice, has been reported in the marketing literature [see e.g. 1, 3, 5, 8-11, 13]. The author presents an iterative least squares method for estimating the parameters of the LLM. In this regression procedure individual consumers represent data points. Their purchase histories constitute the independent variables and actual brand choices are the dependent variables.

First a brief description of the LLM is given. Then the new estimation method is described in some detail. Reports are presented on empirical estimation obtained with the new method for different data sets. Also a goodness-of-fit test is applied to the data. The results of a simulation study, carried out to validate the new estimation method, are given, and the consistency of the procedure is discussed. For the data sets, the LLM paramters were estimated also by the minimum

*Berend Wierenga is a senior lecturer of the Marketing Department of the Wageningen Agricultural University, The Netherlands, who visited Stanford University during $1974 / 75$. This research was supported by grants from Ahold N.V. (the "Albert Heyn Studie Beurs" was awarded to the author) and the Fulbright-Hays Program. Useful comments on a draft of the article by Prof. Srinivasan and doctoral student Shelby McIntyre, both of Stanford University, and comments from $J M R$ referees are gratefully acknowledged. Jaap Bijkerk carried out part of the computer work for this article. chi-square estimation method developed by Massy et al. [10, Chapter 5]. These results are compared with those from the least squares method and some general theoretical and practical aspects of both procedures are discussed.

\section{LINEAR LEARNING MODEL}

The linear learning model for brand choice processes is defined by the following operators [see e.g. 10, Chapter 5; 13, Chapters 3-5]:

$$
p=\alpha+\beta+\lambda p_{t+1} \quad \text { (purchase operator) }
$$

and

$$
p=\alpha+\lambda p_{t-1} \quad \text { (rejection operator). }
$$

The market is assumed to contain only two brands, indicated here as brand 1 and brand $0 . p$, stands for the probability that brand 1 is chosen at purchase occasion $t$. After a purchase, the probability of a consumer purchasing brand 1 is transformed according to equation 1 if brand 1 is chosen; otherwise the transformation is according to equation 2 .

The parameters $\alpha, \beta$, and $\lambda$ are non-negative and because the $p$, are probabilities the constraint

$$
(\alpha+\beta+\lambda) \leq 1
$$

must hold.

The LLM can also be formulated as follows. Let $\left\{X_{l}\right\}$ denote the (stochastic) brand choice process. $X_{t}$ can only take the values 1 and 0 , corresponding with a brand 1 and a brand 0 purchase, respectively. 
Then:

(4) $p_{t}=\operatorname{Prob}\left(X_{t}=1 \mid x_{t-1}, p_{t-1}\right)=\alpha+\beta x_{t-1}+\lambda p_{t-1}$ $\left(x_{t-1}\right.$ is the purchase made at $(t-1)$, which is also either 1 or 0 ).

Thus equation 4 is a complete definition of the LLM and includes equations 1 and 2 . Now according to equation 4:

$$
p_{t-1}=\alpha+\beta x_{t-2}+\lambda p_{t-2} .
$$

Substituting this in equation 4 gives:

$$
p_{t}=\alpha(1+\lambda)+\beta\left(x_{t-1}+\lambda x_{t-2}\right)+\lambda{ }^{2} p_{t-2} \text {. }
$$

Repeating this type of substitution $(k-1)$ times gives:

$$
p_{t}=\alpha \sum_{j=0}^{k-1} \lambda^{j}+\beta \sum_{j=0}^{k-1} \lambda j x_{t-j-1}+\lambda^{k} p_{t-k} .
$$

Equation 7 plays a major role in the estimation method to follow.

\section{LEAST SQUARES ESTIMATION METHOD}

\section{Basic Approach}

Because consumers generally cannot give their current or even past probability of a brand 1 purchase, the LLM parameters cannot be estimated directly from equation 4. Therefore an indirect way is followed that has equation 7 as its starting point. In equation 7 the probability of a brand 1 at purchase occasion $t$ is expressed as a function (with parameters $\alpha, b$, and $\lambda$ ) of (1) the purchase history during the $k$ most recent purchases and (2) the probability of brand 1 purchase at $(t-k)$. Because $0 \leq \lambda \leq 1$ the effect of the latter part (the third term on the right side of equation 7) becomes smaller and smaller as $k$ increases. Only in the extreme case of no learning at all $(\lambda=1)$ is this phenomenon not true. In applications of the LLM the values found for $\lambda$ are mostly within the range .3 to $.7[1 ; 10$, Chapter $5 ; 13$, Chapter 4$]$. Now when $k=10$ for example, one has $(0.3)^{k}=0.000$ and $(0.7)^{k}=0.027$. To obtain the contribution of the last term in equation $7, \lambda^{k}$ has still to be multiplied by $p_{t-k}$, a number between zero and one, which makes it even smaller. So for $k$ that does not have too small a value, the last term in equation 7 is always very small.

Therefore in this section this term is omitted:

$$
p_{c}=\alpha \sum_{j=0}^{k-1} \lambda^{j}+\beta \sum_{j=0}^{k-1} \lambda^{j} x_{t-j-1}
$$

The influence of the remote past of a consumer's purchase history on his current purchase probability is neglected. (In the next section the effect of this remote past is explicitly included.)

After defining

$$
v_{k}(\lambda)=\alpha \sum_{j=0}^{k-1} \lambda^{j}
$$

and

$$
w_{k t}(\lambda)=\sum_{j=0}^{k-1} \lambda x_{t-j-1}
$$

one can write equation 8 as:

$$
p_{t}=v_{k}(\lambda)+\beta w_{k t}(\lambda) \text {. }
$$

Now for the time being the value of $\lambda$ is fixed.

Suppose one has the purchase histories of a sample of $N$ consumers, referring to their last $(k+1)$ purchase. Let the index $t$ of equation 11 correspond with the most recent purchase. Now a consumer's sequence of purchase at $(t-1),(t-2), \ldots,(t-\mathrm{k})$ can be conceived of as his purchase history $x_{t-1}$, $x_{t-2}, \ldots, x_{t-k}$. His most recent purchase at $t$ is the currently observed brand choice. Let the latter purchase be indicated as $y$. Of course $y$ is either 1 or 0 . Different consumers mostly have different purchase histories and for each consumer a value of $w_{k t}(\lambda)$ can be computed with equation 10. So for each consumer $i$ one then has two data points: $y_{i}$ and $w_{k t}(\lambda)$, where $w_{k t}(\lambda)$ depends on $i$. Deleting the subscripts $k$ and $t$ because they are the same for all consumers and remembering that $v$ and $w$ depend on $\lambda$, one can write equation 11 for the individual consumer $i$ as:

$$
p_{i}=v+\beta w_{i}
$$

Now:

$$
E y_{i}=p_{i}
$$

so that

$$
E y_{i}=v+\beta w_{i} .
$$

This is equivalent to:

$$
y_{i}=v+\beta w_{i}+u_{i}
$$

where $E u_{i}=0$.

So with equation 12 one has a regression model in two variables.

Given a series of pairs of observations $\left(y_{i}, w_{i}\right)$, $v$ and $\beta$ can be estimated by the well-known method of least squares [see e.g. 7] in which the sum of the squared deviations between $p_{i}$ and $y_{\mathrm{i}}$ is minimized. Of course $\alpha$ can be derived directly from $v$ with equation 9.

So far the value of $\lambda$ has been fixed so that the squared deviations are minimized for a given value of $\lambda$. Now the least squares procedure is repeated for different values of $\lambda$, until that $\lambda$ has been found for which the overall sum of squared deviations is as small as possible. This is also the $\lambda$ for which the coefficient $R^{2}$, referring to equation 12 , is maximum. Besides estimates for $\alpha, \beta$, and $\lambda$, the least 
squares procedure also gives the value for $R^{2}$ and estimates for the standard deviation of $\alpha$ and $\beta$.'

\section{Bayesian Treatment of Remote Purchase History}

So far the effect of purchases made more than $k$ purchase occasions ago has been neglected. Theoretically the error made by omitting the term on the extreme right of equation 7 can be made as small as desired by making $k$ large enough. However, for large values of $\lambda$ (i.e., large carryover effect), the length of the empirical purchase histories required can become too great for practical estimation. Moreover, the value of $\lambda$ is not known in advance. Therefore in this section the effect of the remote past is taken into account when $k$ is not that big.

Assume that the procedure of the last section has been carried out for a data set, where a particular value for $k$ is chosen. Then the estimates $\hat{\alpha}, \hat{\beta}$, and $\hat{\lambda}$ are available. Now consider a consumer $i$ with a certain purchase history $h_{i}$. (For $k=10,1,024$ different purchase histories are possible). Then given this purchase history and the parameter values $\hat{\alpha}, \hat{\beta}$, and $\hat{\lambda}$, what can be said about this consumer's probability of a brand 1 purchase $k$ purchase occasions ago, i.e. his $p_{t-k}$ ?

The probability of a certain value for this chance is given by Bayes' Theorem:

$$
\operatorname{Prob}\left(p_{t-k} \mid h_{i}\right)=\frac{\operatorname{Prob}\left(h_{i} \mid p_{t-k}\right) \operatorname{Prob}\left(p_{t-k}\right)}{\operatorname{Prob}\left(h_{i}\right)} .
$$

If one assumes that $p_{t-k}$ can only take $r$ different values ranging from $m_{1}$ to $m_{r}$, equation 13 can be written for a particular value, viz. $m_{l}$, as:

$$
\begin{aligned}
\operatorname{Prob}\left(p_{t-k}=\right. & \left.m_{l} \mid h_{i}\right) \\
= & \frac{\operatorname{Prob}\left(h_{i} \mid p_{t-k}=m_{l}\right) \operatorname{Prob}\left(p_{t-k}=m_{l}\right)}{\sum_{d=1}^{r} \operatorname{Prob}\left(h_{i} \mid p_{t-k}=m_{d}\right) \operatorname{Prob}\left(p_{t-k}=m_{d}\right)} .
\end{aligned}
$$

The likelihood of purchase history $h_{i}$ when $p_{t-k}=m_{l}$, i.e.,

$$
\operatorname{Prob}\left(h_{i} \mid p_{t-k}=m_{l}\right)
$$

for the LLM-parameter values $\hat{\alpha}, \hat{\beta}$, and $\hat{\lambda}$ can be computed directly by use of the definition of the LLM. This computation can be done for all $r$ possible values of $p_{t-k}$. Suppose no prior information so that all values

\footnotetext{
'Because the dependent variables are probabilities, generalized least squares could be used instead of ordinary least squares. The author decided not to do this because (1) the true $p$-values with which the weighting must be done are not known and one would have to use estimates instead; (2) consumers with $p$ near one or zero would get a heavier weight than consumers with less extreme $p$ values and this would imply that loyal consumers (either to brand 1 or 0 ) contribute more to the estimation results than consumers who switch more frequently.
}

of $p_{t-k}$ are a priori equally likely, i.e.:

$$
\operatorname{Prob}\left(p_{t-k}=m_{l}\right)=\frac{1}{r}(l=1, \ldots, r) .
$$

Now all quantities in equation 14 are known and the posterior probabilities for the $p_{t-k}$-values-given purchase history $h_{i}$-can be determined. In this way a posterior distribution over the $r$ values of $p_{t-k}$ can be obtained for each consumer.

In the present case it is not necessary to represent the distribution of $p_{t-k}$ very finely, i.e., with a large number of different values. Because in equation 7 $\lambda^{k}$ is small, the contribution of the term $\lambda^{k} p_{t-k}$ is sufficiently determined when only the first digit of $p_{t-k}$ is known. Therefore take $r=10$ and split up the $(0,1)$ interval (the range of $p_{t-k}$ ) in 10 parts of equal size, each represented by its midpoint: 0.05 , $0.15, \ldots, 0.95$. For each of these values the $a$ priori probability is 0.10 .

\section{The Complete Method}

In the complete method, iterative regression neglecting the effect of the remote past is carried out first. This step produces initial estimates for $\alpha, \beta$, and $\lambda$. Then the $p_{t-k}$ distributions are updated in the Bayesian way. From the updated $p_{t-k}$ distribution for each consumer the modus is taken as the value to be inserted in equation 7 . When defining:

$$
z_{k t}(\lambda)=\lambda^{k} p_{t-k},
$$

equation 7 can be written as:

$$
p_{r}=v_{k}(\lambda)+\beta w_{k t}(\lambda)+z_{k r}(\lambda),
$$

which is the same as equation 11 but with the effect of the remote past taken into account. For given $\lambda, z_{k \text {, }}$ $(\lambda)$ is known for each consumer. Deleting the subscripts $k$ and $t$, one obtains from equation 17 for individual consumer $i$ :

$$
p_{i}=v+\beta w_{i}+z_{i}
$$

Now defining:

$$
y_{i}^{*}=y_{i}-z_{i},
$$

estimates of $v$ and $\beta$ can be obtained by means of least squares regression of $y^{*}$ on $w$. This process is repeated for a new value of $\lambda$, etc. So the same iterative procedure as before is used to obtain new estimates for $\alpha, b$, and $\lambda$, the only difference being that $y$ is replaced by $y^{*}$. When the new estimates are close to the old ones, the procedure stops. Otherwise the $p_{t-k}$ distribution is updated once again and the estimation is repeated. So there are two iteration cycles. In the first one the initial $p$-distribution is varied, and in the second one the LLM-parameter $\lambda$ takes different values. The second cycle is carried out within each step of the first one. 
Table 1

LEAST SQUARES ESTIMATION RESULTS FOR THREE FOOD PRODUCTS

\begin{tabular}{|c|c|c|c|c|c|c|c|c|c|}
\hline Product & Brand I & $\hat{\alpha}$ & $\hat{\boldsymbol{\beta}}$ & $\hat{\lambda}$ & $\hat{\sigma}_{\dot{\alpha}}$ & $\hat{\boldsymbol{\sigma}}_{\dot{\boldsymbol{p}}}$ & $\Sigma e_{i}^{2} / n$ & $R^{2}$ & $n$ \\
\hline \multirow[t]{4}{*}{ Beer } & BI & .009 & .242 & .745 & .004 & .007 & .075 & .675 & 613 \\
\hline & B2 & .004 & .251 & .717 & .003 & .009 & .050 & .579 & 613 \\
\hline & B3 & .000 & .234 & .757 & .002 & .005 & .021 & .788 & 613 \\
\hline & B4 & .005 & .322 & .653 & .004 & .008 & .058 & .714 & 613 \\
\hline Fopro & F1 & .018 & .586 & .384 & .006 & .010 & .038 & .840 & 666 \\
\hline Marg & MI & .002 & .243 & .755 & .002 & .004 & .031 & .835 & 850 \\
\hline
\end{tabular}

\section{EMPIRICAL RESULTS}

The estimation method was applied to brand choice data for three frequently purchased consumer products in the Netherlands: beer, fopro (a pseudonym for a food product), and margarine. The purchases were made during 1967 and 1968 by members of the Dutch Attwood Consumer panel. The numbers of households of which the brand choice data were used are 613, 666, and 850 for beer, fopro, and margarine, respectively. More details about the data can be found in [13]. For each product the biggest brand in the market was called brand 1 . For beer, fopro, and margarine these are indicated as $B 1, F 1$, and $M 1$, respectively. All other brands in a market are designated as brand 0 . The beer data also were examined with three other brands in turn indicated as brand 1 . These brands are called B2, B3, and B4, respectively.

For every household the first 10 purchases made in the reporting period are considered to be the purchase history; the eleventh purchase constitutes the subsequently observed brand choice (the $y_{i}$ of the previous sections). So $k$ is taken to be 10 here.

In Table 1 the estimates obtained for $\alpha, b$, and $\lambda$, corresponding standard deviations, the mean square deviation $\left(\Sigma e_{i}^{2} / n\right)$, and $R^{2}$ are presented. Table 2 shows some additional statistics: the correlation coefficient of $\hat{\alpha}$ and $\hat{\beta}$; the ratios coefficient/standard deviation for $\hat{\alpha}$ and $\hat{\beta}$ and lower and upper limits for $p$.

To give an idea about the effect of taking into account the remote part of the purchase history, Table 3 shows the parameter estimates for the first and last iteration rounds described in the previous section. An iteration was terminated when the third digit of $\hat{\lambda}$ did not change from one iteration to the next.
To illustrate the dependence of the sum of squared deviations $\left(\Sigma e_{i}^{2}\right)$ on $\lambda$ within an iteration round, Figure 1 depicts the relationship between these two quantities for the first iteration round of beer, B1. The pattern in Figure 1 is typical for all other cases in that $\Sigma e_{i}^{2}$ always had an unambiguous lowest point without problems of local minima.

\section{Comments}

1. The estimates found for the LLM parameters $\alpha$, $\beta$, and $\lambda$ look reasonable and are in agreement with restriction (3). If this were not so, the fit of the LLM would be questionable here. In the LLM the range of values of $p$ (the probability of a brand 1 purchase) is limited. The lower limit is $p_{L}=\alpha$ $/(1-\lambda)$; the upper limit is $p_{v}=(\alpha+\beta) /(1-\lambda)$. In Table 2 these limits are given for the parameter values of Table 1 . These boundary values look reasonable $-p$ becomes very small after a great many brand 0 purchases and very large after many brand 1 purchases. The probability of a brand 1 purchase never becomes exactly equal to 0 or 1 , however. Theoretically, when there are more than two different brands in a market, the slope $\lambda$ of the LLM should be equal for all brands [see 6]. For beer this condition can be checked. It appears that the estimated $\lambda s$ are rather close together. They range from 0.6528 to 0.7565 , and three of them are within a range of 0.04 . The value for $\mathrm{B} 4$ is the most extreme, probably because B4, unlike all other brands distinguished, is not a real brand in the market, but a conglomerate-i.e., defined as all brands that are not B1, B2, or B3.

2. The standard deviations for the $\hat{\beta}$ s are relatively small. For the $\hat{\alpha} s$ they are large, especially when $\hat{\alpha}$ is small. This result has implications for the accuracy of long-term market shares predictions.

Table 2

ADDITIONAL STATISTICS WITH RESPECT TO THE LLM PARAMETERS, OBTAINED BY LEAST SQUARES METHOD

\begin{tabular}{|c|c|c|c|c|c|c|}
\hline Product & Brand I & $\hat{\rho}(\hat{\alpha}, \hat{\beta})$ & $\hat{\alpha} / \hat{\sigma}_{\alpha}$ & $\hat{\beta} / \hat{\sigma}_{\beta}$ & $P_{L}$ & $\boldsymbol{P}_{u}$ \\
\hline \multirow[t]{4}{*}{ Beer } & BI & -.70 & 2.04 & 35.66 & .033 & .980 \\
\hline & B2 & -.43 & 1.39 & 28.98 & .014 & .901 \\
\hline & B3 & -.38 & .21 & 47.61 & .002 & .961 \\
\hline & B4 & -.59 & 1.10 & 39.07 & .013 & .941 \\
\hline Fopro & FI & -.62 & 2.99 & 59.09 & .029 & .980 \\
\hline Marg & M1 & -.55 & .89 & 65.56 & .007 & .999 \\
\hline
\end{tabular}


Table 3

PARAMETER ESTIMATES FOR FIRST AND LAST ITERATION ROUNDS

\begin{tabular}{|c|c|c|c|c|c|c|c|c|}
\hline \multirow[b]{2}{*}{ Product } & \multirow[b]{2}{*}{ Brand 1} & \multicolumn{3}{|c|}{$\begin{array}{l}\text { First iteration } \\
\text { round }\end{array}$} & \multicolumn{3}{|c|}{$\begin{array}{l}\text { Last iteration } \\
\text { round }\end{array}$} & \multirow{2}{*}{$\begin{array}{c}\text { Number of } \\
\text { rounds } \\
\text { required }\end{array}$} \\
\hline & & $\hat{\boldsymbol{\alpha}}$ & $\hat{\beta}$ & $\hat{\lambda}$ & $\hat{\boldsymbol{\alpha}}$ & $\hat{\boldsymbol{\beta}}$ & $\hat{\lambda}$ & \\
\hline Beer & $\begin{array}{l}\text { B1 } \\
\text { B2 } \\
\text { B3 } \\
\text { B4 }\end{array}$ & $\begin{array}{l}.009 \\
.004 \\
.001 \\
.005\end{array}$ & $\begin{array}{l}.238 \\
.240 \\
.234 \\
.329\end{array}$ & $\begin{array}{l}.765 \\
.743 \\
.774 \\
.649\end{array}$ & $\begin{array}{l}.009 \\
.004 \\
.000 \\
.005\end{array}$ & $\begin{array}{l}.242 \\
.251 \\
.234 \\
.322\end{array}$ & $\begin{array}{l}.745 \\
.717 \\
.757 \\
.653\end{array}$ & $\begin{array}{l}3 \\
3 \\
3 \\
3\end{array}$ \\
\hline Fopro & $\mathrm{F} 1$ & $\begin{array}{r}.018 \\
002\end{array}$ & $\begin{array}{r}.586 \\
225\end{array}$ & .384 & .018 & .586 & .384 & 2 \\
\hline
\end{tabular}

As shown in $[10$, p. 148] the expression for the long-term market share of brand 1 is:

$$
M=\frac{\alpha}{1-\beta-\lambda} .
$$

Here the parameter $\alpha$ plays a crucial role and a large variance of $\hat{\alpha}$ causes a large variance of the predicted long-term market share. There is a negative covariance between $\hat{\alpha}$ and $\hat{\beta}$, clearly related to the tendency of $(\alpha+\beta+\lambda)$ to be near 1 .

3. Table 3 shows that the effect of incorporating the remote part of the purchase history on the estimated values for $\alpha, b$, and $\lambda$ is not great for the data considered here. Sometimes in the first iteration round the restriction (3) is violated, however.

4. The values of $R^{2}$ are generally good. There is a clear influence of the purchase history on current brand choice. However, with the interpretation of $R^{2}$ caution is required. Here the value of $R^{2}$ is not only a function of the goodness of fit of the data to the LLM estimated, but also a function of the particular parameter values of this model itself. In general $\Sigma e_{i}^{2}$ tends to be smaller and $R^{2}$ tends to

Figure I

DEPENDENCE OF $\Sigma e_{i}^{2}$ ON $\lambda$ FOR BEER, BRAND B 1

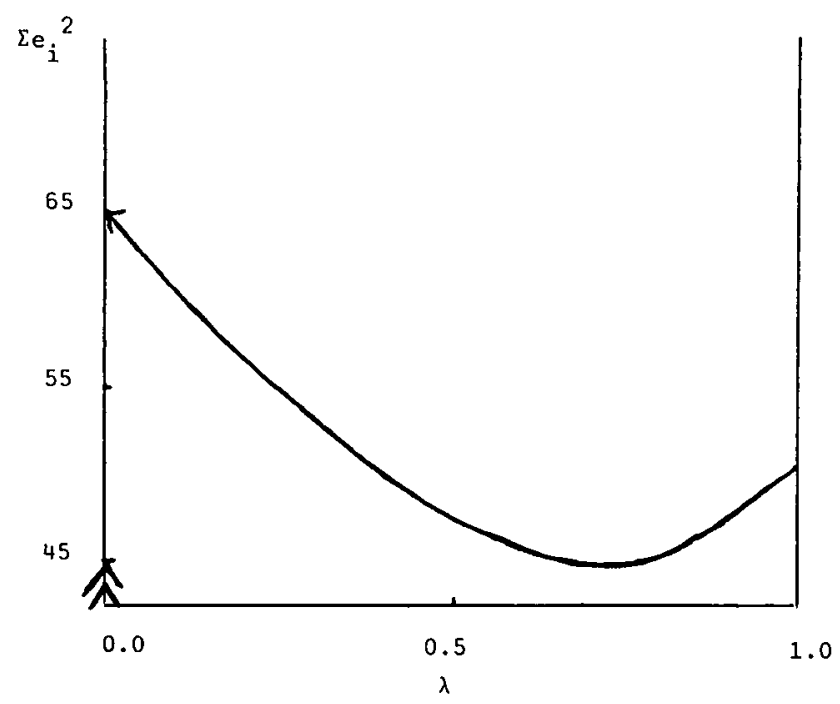

be larger as the particular LLM allows the values of $p$ to become closer to 0 and 1 . (For the case in which $p$ has a beta distribution, the upper bound for $R^{2}$ can be computed; see [12]). Therefore the comparison of $R^{2}$ values for different data sets does not provide a complete picture of the relative goodness of fit of the LLM.

\section{A TEST FOR GOODNESS OF FIT}

Once the LLM parameters are estimated, for each consumer $i$ one can compute the predicted $p_{i}$, i.e., the probability of choosing brand 1 , which can be compared with $y_{i}$, the actual outcome of the brand choice. (Here $p_{i}$ and $y_{i}$ have the meaning from the previous paragraphs). The hypothesis to be checked is:

$$
\operatorname{Prob}\left(y_{i}=1\right)=p_{i}(i=1, \ldots, N) .
$$

A test described by Cox [4, Section 4.4] is used to examine this hypothesis. The test consists of two parts and uses the logistic transform, extensively discussed by Cox. The first part is concerned with the question of whether the $p_{i}$ are systematically too high or too low. The test statistic is:

$$
T_{1}=\Sigma y_{i}
$$

Under the null hypothesis "the $p_{i}$ are neither too high or too low," the statistic

$$
S_{1}=\frac{T_{1}-E T_{1}}{\sqrt{\operatorname{Var}} T_{1}}
$$

is distributed approximately normally.

The second part of the test is concerned with the question of whether the $p_{i}$ are too clustered or too dispersed. Here is a test statistic $T_{2}$ is used, which after transformation into

$$
S_{2}=\frac{T_{2}-E T_{2}}{\sqrt{\operatorname{Var}} T_{2}}
$$

is distributed approximately normally under the hypothesis: "the $p_{\text {, }}$ are neither too clustered nor too dispersed." When the $p_{i}$ are too dispersed, $T_{2}$ is smaller than expected under the null hypothesis; when the 
$p_{i}$ are too clustered, $T_{2}$ is larger. For the definition of $T_{2}$ and more information about these tests the reader is referred to Cox [4].

Results obtained with these tests for the author's data are presented in Table 4 . From the low values in this table it appears that the $p_{i}$ predicted by the LLM, using the least squares parameter estimates, perform very well. None of the $p_{i}$ values is too low or too high, or too clustered or too dispersed. ${ }^{2}$

\section{VALIDATION OF THE PROCEDURE AND CONSISTENCY OF THE ESTIMATORS}

\section{Simulation Study}

To examine how well the least squares estimation method reproduces the true LLM parameters, a simulation study was run in which the parameters were estimated from purchase histories generated by LLM brand choice processes with known true parameters. Three different parameter sets were used, the "classic" Snow Crop case [10, p. 172-5] and the cases of $B 1$ and F1.

First the situation of 500 households and a history of five purchases per household was considered. Then the effects of increasing the number of households to 1,000 and increasing the number of purchases to 10 were examined. The results for the estimated parameter values are summarized in Table 5. For each combination in this table 10 simulation runs (with different random starting values) were carried out. For individual households the probabilities of choosing brand 1 at the first purchase were obtained as drawings from beta distributions corresponding to the respective parameter sets (the $\mu_{1}$ and $\mu_{2}$ values given in [10, Table 5.5.] for Snow Crop and in [13, Table 4.23] for beer and fopro were used to determine the beta parameters). Table 5 shows that the reproduction of the original parameters is satisfactory. In the case

Table 4

RESULTS OF COX TEST FOR GOODNESS OF FIT OF LLM, LEAST SQUARES ESTIMATED PARAMETERS

\begin{tabular}{lcccc}
\hline \hline Product. & Brand I & $S_{1}$ & $S_{2}$ & $n$ \\
\hline Beer & B1 & -.00 & .00 & 613 \\
& B2 & -.02 & -.13 & 613 \\
& B3 & -.03 & -.46 & 613 \\
Fopro & B4 & -.01 & -.05 & 613 \\
Marg & F1 & -.01 & -.04 & 666 \\
& M1 & -.03 & -.37 & 850
\end{tabular}

\footnotetext{
${ }^{2}$ The $p_{i}$ used in the test are not given a priori but are computed from parameter estimates obtained from the data. Strictly speaking, the variance of the test statistic should be corrected for errors in these estimates. Because such a correction generally would increase this variance, the resulting values of $S_{1}$ and $S_{2}$ would become even smaller, thus strengthening the conclusion.
}

of 500 households and five purchases per household the mean error ranges from .008 to .096 .

The last line in Table 5 gives an impression about possible biases in the estimator. Because 30 simulations were run for each parameter set (10 for each $N-N N$ combination), for an unbiased estimator one would expect 15 estimated parameter values to be under the true values. Table 5 shows that the observed numbers of underestimates are close to this expected number with the exception of $\hat{\alpha}$ for parameter set B. This value was systematically too low, probably because of the small true value of $\alpha$ in this parameter set $(<0.01)$.

\section{Consistency of the Estimators}

In terms of the symbols of equation 12 the true relationship between current purchase and purchase history is:

$$
y_{i}=v+\beta w_{i}+\lambda^{k} p_{i-k}^{i}+u_{i}^{T}
$$

where $u_{i}^{T}$ is the true error term for consumer $i$. In the first iteration round of the estimation procedure, however, the model is specified as:

$$
y_{i}=v+\beta w_{i}+u_{i} \quad \text { (= equation 12) }
$$

Thus the error term in this equation- $-u_{i}-$ is composed of the true error term and a term dependent on $p_{t-k}$, i.e.,

$$
u_{i}=u_{i}^{T}+\lambda^{k} p_{i-k}^{i}
$$

Now according to equation 12

$$
\left.p_{r-k}^{i}=\left(x_{i-k}^{i}-u_{i-k}^{i}\right) \quad \text { (because by definition } y_{i}^{i}=x_{i}^{i}\right) \text {, }
$$

and according to equation 10

$$
w_{i}=\sum_{j=0}^{k-1} \lambda x_{i-j-1}^{i}
$$

So in equation $12 x_{t-k}$ appears both in $w_{i}$ and in the error term $u_{i}$. Because for consistent estimators these quantities ought to be uncorrelated, the question is whether this dependence causes a consistency bias in the estimates obtained by the procedure proposed here. The following remarks can be made.

First, for $k \rightarrow \sim$ the discrepancy between equations 12 and 21 disappears, so the procedure is consistent in the sense that when the length of the observed purchase histories increases the estimates converge in probability to their true values. But increasing the number of households, keeping the length of the purchase history fixed, will not automatically remove the dependence. Second, even for short purchase histories the impact of the correlation between $w_{i}$ and $u_{i}$ can be assumed to be very small. The term $x_{\text {. }}$ is only one of the components of $w_{i}$ and in fact the component with the smallest weight $\left(\lambda^{k-1}\right.$, see equation 10). Third, in the second round (and in all additional rounds) of the iteration procedure the term 
Table 5

SIMULATION RESULTS FOR THREE PARAMETER SETS

\begin{tabular}{|c|c|c|c|c|c|c|c|c|c|}
\hline & \multicolumn{3}{|c|}{$\begin{array}{c}\text { Parameter set } A: \\
\text { Snow Crop } \\
\alpha=.015 \\
\beta=.305 \\
\lambda=.612\end{array}$} & \multicolumn{3}{|c|}{$\begin{array}{c}\text { Parameter set } B: \\
\text { beer } B I \\
\alpha=.009 \\
\beta=.242 \\
\lambda=.745 \\
\end{array}$} & \multicolumn{3}{|c|}{$\begin{array}{c}\text { Parameter set } C \text { : } \\
\text { fopro } F \mid \\
\alpha=.018 \\
\beta=.586 \\
\lambda=.384\end{array}$} \\
\hline & $\hat{\alpha}$ & $\hat{\boldsymbol{\beta}}$ & $\hat{\lambda}$ & $\hat{\alpha}$ & $\hat{\boldsymbol{\beta}}$ & $\hat{\lambda}$ & $\hat{\alpha}$ & $\hat{\boldsymbol{\beta}}$ & $\hat{\lambda}$ \\
\hline $\begin{array}{l}N=5 \\
N N=500\end{array}$ & \multicolumn{3}{|c|}{. } & & & & & & \\
\hline MES & .012 & .328 & .586 & .008 & .283 & .704 & .016 & .597 & .380 \\
\hline MER & .008 & .045 & .063 & .009 & .059 & .067 & .008 & .087 & .096 \\
\hline MSE & .008 & .062 & .083 & .010 & .080 & .089 & .009 & .099 & .107 \\
\hline \multicolumn{10}{|l|}{$\begin{array}{l}N=5 \\
N N=1,000\end{array}$} \\
\hline MES & .012 & .313 & .612 & .004 & .268 & .727 & .020 & .620 & .347 \\
\hline MER & .005 & .025 & .026 & .006 & .040 & .044 & .005 & .067 & .073 \\
\hline MSE & .006 & .030 & .033 & .006 & .048 & .059 & .006 & .091 & .101 \\
\hline \multicolumn{10}{|l|}{$\begin{array}{l}N=10 \\
N N=500\end{array}$} \\
\hline MES & .019 & .321 & .594 & .007 & .204 & .787 & .016 & .600 & .374 \\
\hline MER & .005 & .021 & .038 & .002 & .039 & .043 & .007 & .081 & .087 \\
\hline MSE & .006 & .028 & .042 & .003 & .053 & .059 & .007 & .100 & .111 \\
\hline $\begin{array}{l}\text { Number of times } \\
\text { estimated parameter } \\
\text { value is equal to or } \\
\text { below true value }\end{array}$ & 15 & 12 & 16 & 22 & 16 & 13 & 15 & 14 & 15 \\
\hline $\begin{array}{l}N=\text { length of obser } \\
N N=\text { number of ho } \\
M E S=\text { mean estima } \\
M E R=\text { mean error. } \\
M S E=\text { mean squar } \\
\text { Number of simulatic }\end{array}$ & $\begin{array}{l}\text { chase } \\
\text { is in } t\end{array}$ & ry. & le. & & & & & & \\
\hline
\end{tabular}

$\lambda^{k} p_{i-k}^{i}$ is restored. Although the $p_{t-k}^{i}$ s used here are approximations of the true values, the consistency problem is considerably reduced.

Table 6 illustrates these points. For simulated LLM purchase histories (for which the true $p_{t-k}^{i}$ values were known) the mean value of the product of error term and $w_{i}$ was calculated for (1) the true error term $u_{i}^{T}$, (2) the error term $u_{i}$ in the first iteration round, and (3) the error term $u$ in the second iteration round.

The specific LLM parameters used in the simulation are the Snow Crop values: $.015, .305$, and .612. The effect of the dependence in the error term can be assessed by the extent to which the product $\Sigma u_{i} w_{i}$ differs more from zero in the case of dependent error terms than in the situation of the true error terms. (In the latter case the expected value is zero.)

As is clear from Table 6 the impact of the dependence is very small, even when the length of the purchase history is only five. Moreover, the dependence effect decreases quickly as $N$ (the length of the purchase history) increases. Also the effect is much less severe in the second than in the first iteration round.

These results are in agreement with the good quality of the estimates in the simulation study. Therefore

Table 6

IMPACT OF CONSISTENCY BIAS FOR SIMULATED PURCHASE HISTORIES

\begin{tabular}{|c|c|c|c|c|c|c|}
\hline & & $\begin{array}{c}(1) \\
\Sigma u_{i}^{T} w_{i} \\
\end{array}$ & $\left|\begin{array}{c}(2) \\
\Sigma u_{i} w_{i}\end{array}\right|$ & $\left|\begin{array}{c}(3) \\
\Sigma u_{i} w_{i}\end{array}\right|$ & & \\
\hline & & $N N$ & $|N N|$ & $\mid N N$ & & \\
\hline$N$ & $N N$ & & $\begin{array}{l}\quad \text { first } \\
\text { iteration round }\end{array}$ & $\begin{array}{l}\text { second } \\
\text { iteration round }\end{array}$ & $(2)-(1)$ & (3) $-(1)$ \\
\hline 5 & 500 & .0240 & .0435 & .0200 & .0195 & -.0040 \\
\hline 10 & 500 & .0119 & .0136 & .0115 & .0017 & -.0004 \\
\hline 15 & 500 & .0109 & .0108 & .0109 & -.0001 & .0000 \\
\hline 5 & 1000 & .0021 & .0141 & .0102 & .0120 & .0081 \\
\hline 10 & 1000 & .0053 & .0066 & .0047 & .0013 & .0006 \\
\hline
\end{tabular}


the consistency issue is not much of a problem, although one should keep in mind that the purchase histories should not be taken too short. (As a guideline: five purchases still gave satisfactory results in this study.)

\section{COMPARISON OF THE LEAST SQUARES METHOD WITH THE MINIMUM CHI SQUARE METHOD}

The most prominent estimation method for the LLM parameter is the minimum chi square procedure developed by Massy et al. [10, Chapter 5]. It was used extensively in [10], and other authors have used the method subsequently [e.g. 1, 9, 11, 13] . Carman [3] described an estimation method which is essentially a regression procedure, but very different from the least squares method presented here. Carman's procedure does not take into account the fact that different consumers will have different $p$-values at the beginning of the observation period.

Haines [5] used various procedures to estimate the LLM parameters. However, he dealt with a simplified version of the LLM and also made the assumption that at the start of the observation period all consumers have the same $p$-value. Here only the minimum chi square method is considered further. This is the first method whereby-in an ingenious way- the heterogeneity of the consumer population with respect to $p$ at the start of the observation period is taken fully into account. Each consumer is assumed to have his own initial $p$-value $p_{0}$, and $p_{0}$ has a distribution in the consumer population with density function $f\left(p_{0}\right)$ and first four moments $\mu_{1}, \mu_{2}, \mu_{3}$, and $\mu_{4}$.

The input for the procedure consists of the relative frequencies with which the 16 different purchase sequences of length four occur in observed purchase histories. According to the theory of the LLM the expected values of these 16 relative frequencies are a function of the LLM parameters $\alpha, \beta$, and $\lambda$ and the four moments of the $p_{0}$ distribution $\mu_{1}$ to $\mu_{4}$. The procedure searches for those values of $\alpha, \beta, \lambda$, $\mu_{1}, \mu_{2}, \mu_{3}$, and $\mu_{4}$ that minimize the differences between observed and expected relative frequencies in a chi square sense. The method is described in detail in [10, Chapter 5].
In the following discussion the minimum chi square method (MCS) is compared with the least squares (LS) procedure.

In [13] the LLM parameters for the beer, fopro, and margarine data which were used here to apply the LS method have been estimated by the minimum chi square method, where the first 10 purchases of each household made in the reporting period are used as observations. The resulting estimates of the LLM parameters (indicated by a $\sim$, in contrast to the LS estimates which are indicated by $a^{~}{ }^{\circ}$ ) are reproduced in Table 7.

By use of the Bayesian procedure described previously, for each household the probability of a brand 1 purchase after the 10 observed purchases can be computed for the LLM parameter estimates $\bar{\alpha}, \tilde{\beta}$, and $\tilde{\lambda}$. These computed $p$-values can be compared with actual brand choices at the eleventh purchase, so that the mean square deviation $\left(\Sigma e_{i}^{2} / n\right)$ and the test statistics $S_{1}$ and $S_{2}$ can be calculated in the same way as for the LS estimates. These also are given in Table 7. The following comments can be made.

1. From the $x^{2}$ values it appears that according to the MCS criterion the fit of the LLM is satisfactory for beer and fopro. For margarine the fit is less satisfactory.

2. The MCS estimates are to be compared with the LS estimates of Table 1. The estimated values for $\alpha$ are small in both tables. Moreover, as seen before, the variance of these estimates is relatively large.

Therefore and because of the phenomenon that estimates of $\beta$ and $\lambda$ are approximately complements of each other (caused by the tendency of $\alpha, \beta$, and $\lambda$ to sum to unity), a comparison of the estimated values of $\lambda$ found by both methods is most appropriate. In three cases $\lambda$ and $\bar{\lambda}$ are rather close together (difference $<.1$ ); in three other cases the differences are somewhat bigger, although never more than 0.2 . Such differences are not very large when one considers the general properties of minimum chi square estimates, especially with respect to their variance [see 2]. For the biggest difference in $\lambda$-values, viz. beer, B3, the value of the chi square criterion was computed using the LS parameter estimates instead of the MCS estimates. Because the LS method produces no corresponding values for $\mu_{1}$ to $\mu_{4}$, these values must be guessed. After some trial and error,

Table 7

MINIMUM CHI SQUARE PARAMETER ESTIMATES AND ADDITIONAL STATISTICS

\begin{tabular}{lccccccccc}
\hline \hline Product & Brand 1 & $\tilde{\alpha}$ & $\bar{\beta}$ & $\bar{\lambda}$ & $\chi_{8}^{2}$ & $\hat{\lambda}-\bar{\lambda}$ & $\Sigma e_{i}^{2} / \mathrm{n}$ & $S_{1}$ & $S_{2}$ \\
\hline Beer & B1 & .017 & .403 & .562 & 6.20 & .183 & .079 & .05 \\
& B2 & .003 & .285 & .690 & 6.20 & .027 & .060 &. .61 \\
& B3 & .001 & .424 & .555 & 4.98 & .202 & .023 & -1.45 \\
& B4 & .008 & .398 & .572 & 5.97 & .081 & .059 & -.72 & -1.80 \\
Fopro & F1 & .005 & .439 & .545 & 2.67 & -.161 & .039 & 1.60 & -1.12 \\
Marg" & M1 & .001 & .168 & .831 & 23.35 & -.075 & .031 & -.08 & 1.37 \\
\hline
\end{tabular}

'The results for margarine in [13] are somewhat different from those given here, because the former ones were based on the first 20 purchases of each consumer instead of 10 . 
values of $\mu_{1}$ to $\mu_{4}$ were found which, combined with the estimates $\hat{\alpha}, \hat{\beta}$, and $\hat{\lambda}$, produced a chi square value for B3 as low as 6.72. Thus for B3 the LS estimates are practically as good as the MCS estimates when the value of the MCS criterion is considered. As already noted, theoretically the values of $\lambda$ for different brands in the same market have to be equal. Thus the smaller range of $\lambda$ values for the four beer brands when LS estimates are compared with MCS estimates (0.10 versus 0.15$)$ is a point in favor of the former. A comparison of $\Sigma e_{i}^{2} / n$ in Tables 7 and 2 shows that the mean square deviations for the MCS estimates, although always slightly higher, are surprisingly near to those for the LS estimates.

3. The test statistics $S_{1}$ and $S_{2}$ in Table 7 are always larger (absolutely) than the corresponding values for the LS estimates in Table 4. For fopro the MCS method tends to produce $p$-values which are systematically too high (the probability of finding a higher value for $S_{1}$ under $\mathrm{H}_{0}$ is $3.5 \%$ ). Although in the other cases individual test statistics are further removed from significance, the better fit for the least square estimates on the whole is evident. Also theoretically the LS method has advantages over the MCS method. With the MCS method when more purchase sequences from the same household are taken as observations, these observations are not independent as they should be. Moreover, the distribution of $p_{0}$ (the probability of choosing brand 1 at the start of an observed purchase sequence) is generally not constant when more sequences from the same consumer are used. Another consideration is the practical point that in the MCS method a highly nonlinear function in seven unknowns must be minimized, whereas in the LS method only three parameters must be estimated, by a straightforward regression procedure, complemented with a simple routine for updating the initial $p$-values of the households. The LS method also can be used to extend the linear learning model. For example, it enables the user to handle an LLM model for brand choice with different operators in different stores.

\section{SUMMARY}

The purpose of this article is to present a quick and efficient method for estimating the parameters of the linear learning model. The least squares method uses purchase histories of individual consumers as input. The method is demonstrated for empirical brand choice data from three different data sets. Reasonable parameter estimates were obtained, which obeyed the constraints of the linear learning model. In a test for goodness of fit these estimates produced very good results. A simulation study to validate the new estimation method also gave a favorable outcome.

Empirical findings for the least squares method were compared with those obtained by the minimum chi square method from the same data. The parameter estimates from the two methods were close in three cases (differences $<0.1$ ) and the differences were somewhat bigger in the three other cases examined (between 0.1 and 0.2 ). In the goodness-of-fit test the minimum chi square estimates did not perform as well as the least squares estimates.

\section{REFERENCES}

1. Aaker, D. A. and J. M. Jones. "Modelling Store Choice Behavior,' Journal of Marketing Research, 8 (February 1971), 38-42.

2. Blattberg, R. C. and S. K. Sen. "An Evaluation of the Application of Minimum Chi-Square Procedures to Stochastic Models of Brand Choice," Journal of Marketing Research, 10 (November 1973), 421-7.

3. Carman, J. M. "Brand Switching and Linear Learning Models," Journal of Advertising Research, 6 (June 1966), 23-31.

4. Cox, D. R. The Analysis of Binary Data. London: Methuen \& Co., 1970.

5. Haines, G. H., Jr. Consumer Behavior, Learning Models of Purchasing. New York: The Free Press, 1969.

6. Herniter, J. D. and R. A. Howard. "Stochastic Marketing Models," in Progress in Operations Research Vol. 2. New York: John Wiley and Sons, Inc., 1964.

7. Johnston J. Econometric Methods, 2nd ed. New York: McGraw-Hill Book Company, 1972.

8. Kuehn, A. A. "Consumer Brand Choice-A Learning Process?" Journal of Advertising Research, 2 (December 1962), 10-7.

9. Lilien, G. L. "Application of a Modified Linear Learning Model of Buyer Behavior," Journal of Marketing Research, 11 (August 1974), 279-85.

10. Massy, W. F., D. B. Montogomery, and D. G. Morrison. Stochastic Models of Buying Behavior. Cambridge, Massachusetts: MIT Press, 1970.

11. McConnel, D. "Repeat Purchase Estimation and the Linear Learning Model," Journal of Marketing Research, 5 (August 1968), 304-6.

12. Morrison, D. G. "Upper Bounds for Correlation Between Binary Outcomes and Probabilistic Predictions," Journal of the American Statistical Association, 67 (March 1972), 68-70.

13. Wierenga, B. An Investigation of Brand Choice Processes. Rotterdam: Rotterdam University Press, 1974. 
Copyright of Journal of Marketing Research (JMR) is the property of American Marketing Association and its content may not be copied or emailed to multiple sites or posted to a listserv without the copyright holder's express written permission. However, users may print, download, or email articles for individual use. 